\title{
AUTOMATIC MODEL VERIFICATION FOR SEMICONDUCTOR MANUFACTURING SIMULATION
}

\author{
Boon Ping Gan \\ Peter Lendermann \\ 8 Jurong Town Hall Road \\ \#30-04 JTC Summit \\ Singapore 609434, SINGAPORE
}

\author{
Wolfgang Scholl \\ Marcin Mosinski \\ Infineon Technologies Dresden \\ Koenigsbruecker Strasse 180 \\ Dresden 01099, GERMANY
}

\author{
Patrick Preuss \\ D-SIMLAB Technologies GmbH \\ Wiener Platz 6 \\ Dresden 01069, GERMANY
}

\begin{abstract}
Short Term Simulation (STS) that provides daily forecasts of work center performance has been deployed in Infineon Technologies for operational decision makings. To ensure good forecast accuracy, the STS requires high modeling fidelity, requiring good basic data quality for model building. Forecast accuracy is maintained through an Automatic Model Verification (AMV) engine. The AMV monitors and verifies discrepancies between simulation and reality for modeling elements such as process dedication, uptime, process time/throughput, sampling rate, and batch/stream size. It reports the verification results with a multi-layered view, at different levels of abstraction, and the gaps between simulation and reality are highlighted. The user can quickly identify gaps and make correction to the errors. In this paper, we give an insight to the complete workflow on how AMV helps to detect data issues, the options to resolve such issues and the positive effect to the simulation forecast quality.
\end{abstract}

\section{INTRODUCTION}

The D-SIMCON Forecaster is a simulation-based software solution that enables proactive Work-InProgress (WIP) management in a semiconductor manufacturing facility by providing a 7-14 day shortterm simulation forecast of wafer arrivals, wafer departures and WIP, with daily granularity and down to work center and product group level (D-SIMLAB 2013). The solution has been deployed in Infineon Technologies (Dresden) for daily operational decision support (Scholl et al. 2010). To achieve good forecast quality, a high-fidelity representation of manufacturing operations is essential. However, in the environment of a complex wafer fabrication facility availability of good quality and up-to-date input data for the simulation model is always a challenge. As such, an Automatic Model Verification (AMV) module is integrated with the D-SIMCON Forecaster to ensure that data quality is maintained, "garbage-in-garbageout" situations are avoided, and data used in simulation actually represents reality well enough. The AMV module primarily verifies data used to drive modeling features affecting production capacity such as uptime, process time, throughput, batch size and stream size, equipment dedication and dispatch rules. 


\section{OVERVIEW}

The short-term simulation model generated by the D-SIMCON Forecaster is an orchestration of many modeling features each of which is crucial for representing semiconductor manufacturing operations. Obviously it should be driven by data and logic that represents reality. Any deviation from reality could lead to degrading forecast quality. It is therefore crucial that the correctness of critical modeling features is continuously monitored. The AMV module is therefore run daily to inspect the simulation model that was generated 7-14 days ago. The verification can only occur after the time has passed since the AMV compares forecasted results and real historical values.

Model verification is carried out by comparing the respective lot traces and equipment state traces in simulation and reality. Any discrepancy detected with regard to one of the above-mentioned modeling features is raised by the AMV module as an issue requiring user intervention to make adjustments. Those issues could be:

- Mismatching tool count.

- Mismatching process dedication to equipment.

- Any deviation of total and daily uptime above a threshold percentage. The threshold percentage is determined based on the significance of an unavailable event towards the work center performance. The more significant the effect, the lower the threshold percentage.

- Deviation of average process time or throughput above a threshold percentage for each combination of equipment, process, and product.

- Discrepancy in both maximum and average batch size at each batch tool.

- Stream size and sampling rates above a certain threshold percentage, respectively.

\section{VERFICIATION ELEMENTS}

In this section, we discuss the modeling elements that are being verified by the AMV. These elements were chosen as any significant deviation will have negative influence on the forecast results.

\subsection{Tool Count}

Production equipment in the fab is grouped into work centers, i.e. logical groups of tools with similar process capabilities. The number of tools assigned to a work center defines the capacity constraint for the processes. Any discrepancy in tool count, i.e. incorrect number of tools assigned to work centers, leads to an incorrect capacity representation.

\subsection{Tool Dedication}

Each tool in the fab is qualified to run certain processes. In reality not all dedications are made use of though. As the simulation is driven by the full dedication list this could result in a discrepancy in tool dedication and therefore process capacity allocation.

\subsection{Work Center Uptime}

Work center uptime is defined as the available productive time. Tool downs, Preventive Maintenance $(\mathrm{PM})$, as well as engineering/prototyping reduce this availability. Tool downs are random events that cannot be predicted. Their effect on work center performance depends on the workload and total capacity availability at the work center: The closer the workload is to the available capacity the more a tool down event will influence the work center performance. PM and engineering/prototyping activities are planned activities but in reality are typically executed with deviation from the plan due to factors such as maintenance personnel availability. As the simulation model is driven by PM and engineering/prototyping plans deviations from plan can affect forecast quality. 


\subsection{Process Time}

Gan, Lendermann, Preuss, Scholl, and Mosinski

Each lot goes through one or several steps within a tool with a defined process time for each step (excluding the time spent on queuing). Process time is typically maintained manually and thus incomplete. Some process time values are thus derived by extrapolating process time values from similar processes, and could be a source of discrepancy. Incorrect process time value being used will cause over or under capacity consumption by a process, and thus invalidate the cycle time representation in the simulation model.

\subsection{Throughput}

Each equipment produces wafers lots with a defined throughput (wafers per hour), constrained by the bottleneck process within a tool. Similar to process time, throughput data could be incomplete and thus derived by extrapolating throughput values from similar processes. Incorrect throughput value being used will cause an invalid capacity representation.

\subsection{Batch Size}

In a wafer fab, furnaces are typical batch tools as they process multiple lots concurrently, depending on the physical setup. In this setting, critical metrics to be verified are not only maximum batch size but also average batch size as batch tools are typically operated with an associated batching policy. The batching policy defines three operating rules:

- The minimum lot size that must be formed before processing can start

- The maximum time to wait for maximum lot size to be formed before processing can start, and

- The maximum time to wait for minimum lot size to be formed before processing can start.

These rules require parameter settings which could possibly cause discrepancies between simulation and reality.

\subsection{Stream Size}

Stream size is defined as the minimum number of lots to be processed at a particular equipment before a setup change, represented by a dispatch rule that holds back such a setup change until the equipment has processed a certain number of lots, unless a certain maximum time limit has expired. A difference in stream size between simulation and reality typically points to an issue with regard to the representation of the respective dispatch rule in the simulation model. As a result the average number of setup changes over time at the respective work center would be inconsistent, and the work center capacity would be different in simulation and reality.

\subsection{Sampling Rate}

In a wafer fabrication manufacturing process, a process route defines all the operation steps that a lot would go through. Some steps are executed by all lots, while some other steps are executed for a subset of lots only. In the simulation model, the latter case is modeled using sampling rates, i.e. probabilities that a lot will go through that operation step. It is computed from historical data of the previous weeks, and fed to the simulation as input data.

\section{RESULT REPRESENTATION}

The objective of the AMV module is to allow the user of the D-SIMCON Forecaster to quickly identify data discrepancies between simulation and reality that could lead to degradation of forecast quality. Moreover, it enables the user to focus on the most critical issues first before addressing less critical ones. As such the AMV presents the results of its analysis in a hierarchical manner with a 3-level data granularity. 


\subsection{AMV - Level 1}

The AMV Level 1 gives an overview of the modeling feature data deviation for all work center in a twodimensional matrix, as illustrated in Figure 1 . The user can quickly identify the work center that has the most significant problem for each modeling feature. The significance of the problem for each work center-modeling feature combination is calculated using the formula as shown below:

$$
\text { Deviation }_{\text {Workcenter,Feature }}=\frac{\sum_{i=1}^{n}\left(\mid \text { FeatureValue }_{i}^{\text {Sim }}-\text { FeatureValue }_{i}^{\text {Real }} \mid\right)}{n}
$$

where $i$ is the equipment belonging to the work center and $n$ is the total number of equipment for the work center.

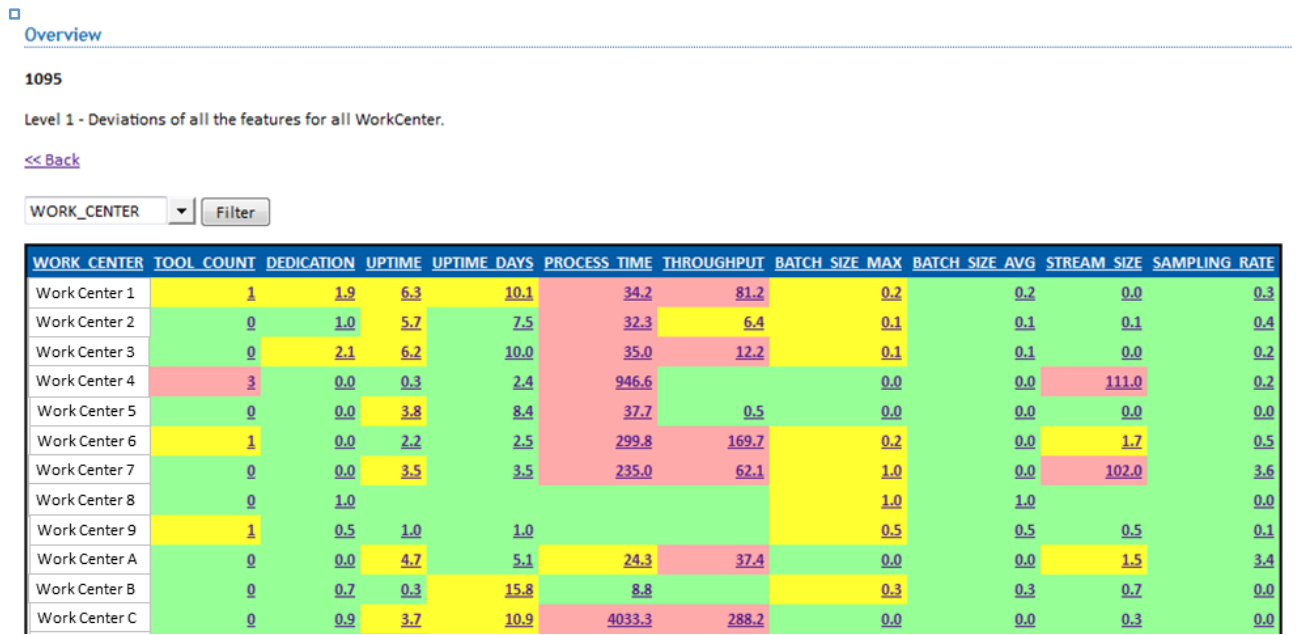

Figure 1: AMV Level 1 - Overview.

The significance of the issue is displayed using a traffic-light color scheme. The traffic-light color is determined by assessing the criticality (importance) of the work center towards the fab capacity (a bottleneck work center would have high criticality/importance) and significance of deviation for the said modeling feature as illustrated in Figure 2. Green color means low deviation independent of the importance of the work center, or a less important work center with medium deviation significance. Yellow color indicates a medium to high importance work center with low to medium deviation significance. A red color indicates a highly important work center with a high deviation significance. The threshold value for the color scheme of the work center importance is derived from its typical capacity utilization, while the threshold value for the color scheme of the deviation significance is derived from modeling feature requirements.

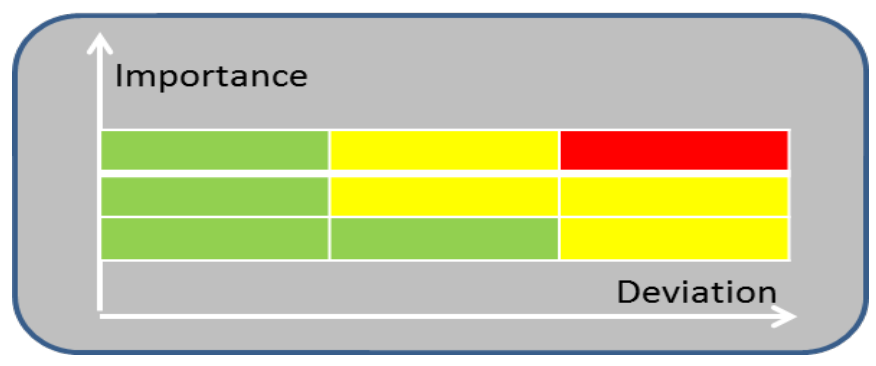

Figure 2: AMV Level 1 - Color Code Definition. 


\subsection{AMV - Level 2}

Gan, Lendermann, Preuss, Scholl, and Mosinski

For each work center and modeling feature combination, the user can navigate to the $A M V$ Level 2, i.e. the next level of detail, to investigate the source of the discrepancy. At this level, the AMV only displays differences between simulation and reality. Figure 3 gives examples of tool dedication and batch size deviation for a work center. For the tool dedication modeling feature, process EPA-1 was executed in the chosen work center for reality, but no moves were observed in simulation. For the batch size modeling feature, the maximum batch sizes for EQUIPMENT-1 and EQUIPMENT-3 are not correctly represented in simulation as the value was 4 lots in reality but only 6 lots in simulation for the former, and the value was 6 lots in reality but 2 lots in simulation for the latter. At this level of detail, a user will be able to pinpoint the processes or equipment that are contributing towards the discrepancy.

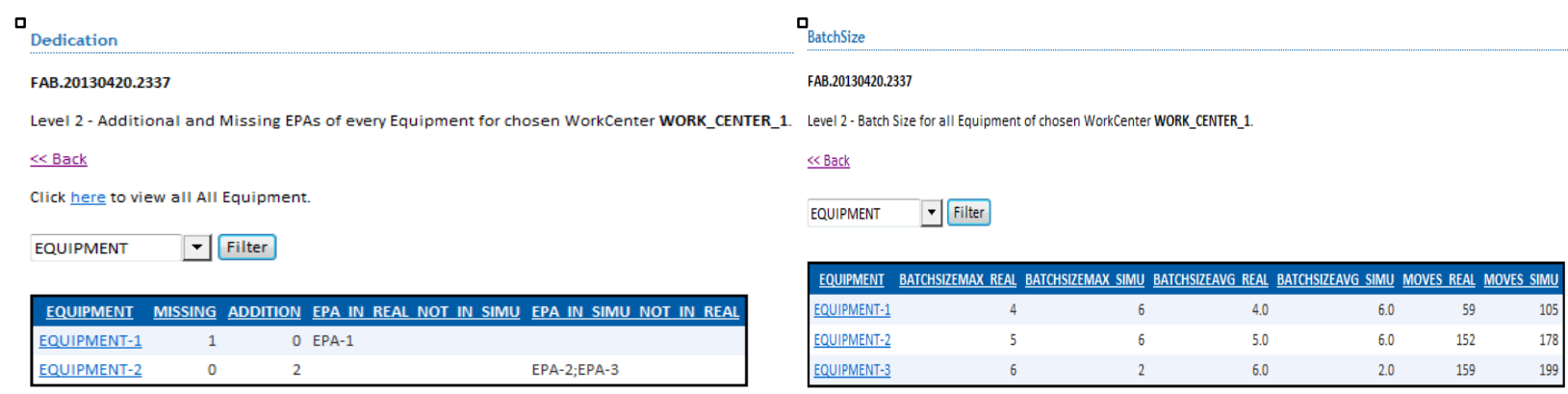

Figure 3: AMV Level 2 - Illustration of Data Discrepancy (Dedication / Batch Size).

\subsection{AMV - Level 3}

The lowest hierarchy level - AMV Level 3 - provides the user the complete data associated with the work center and modeling feature combination. Figure 4 shows a Level 3 tool dedication data presentation. The number of moves in simulation closely approximates the reality for all the eight processes (EPA-1 to EPA-8) processed by the equipment. By adopting this presentation it is very easy for the user to identify the problematic processes by just looking at the chart.

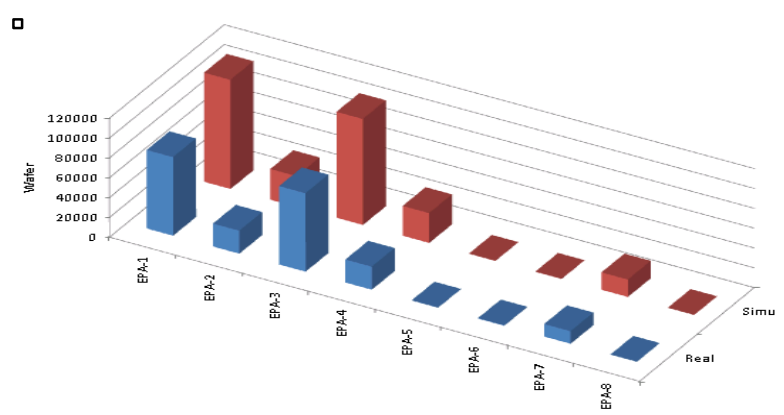

Figure 4: AMV Level 3 - Illustration of Data Discrepancy (Dedication).

A summary of the data presented at the different AMV hierarchy levels for all modeling features is listed in Table 1. 
Gan, Lendermann, Preuss, Scholl, and Mosinski

Table 1: Summary of AMV Verification Hierarchy.

\begin{tabular}{|c|l|l|l|}
\hline Module & \multicolumn{1}{|c|}{ View Level 1 } & \multicolumn{1}{|c|}{ View Level 2 } \\
\hline Tool Count & $\begin{array}{l}\text { Present incorrectly assigned } \\
\text { Equipment for each Workcenter }\end{array}$ & $\begin{array}{l}\text { List of incorrectly assigned Equipment } \\
\text { for chosen Workcenter }\end{array}$ & $\begin{array}{l}\text { List out all Equipment (and their } \\
\text { moves) of selected Workcenter }\end{array}$ \\
\hline Up Time & $\begin{array}{l}\text { Compare Down Time (in percentage) } \\
\text { by Workcenter }\end{array}$ & $\begin{array}{l}\text { Display Down Time and its components } \\
\text { for all Equipment of desired Workcenter }\end{array}$ & $\begin{array}{l}\text { Extend Equipment's Down Time to } \\
\text { each day of the simulation }\end{array}$ \\
\hline Process Time & $\begin{array}{l}\text { Show Process Time deviation per } \\
\text { Workcenter }\end{array}$ & $\begin{array}{l}\text { Process Time for all Equipment-EPA } \\
\text { combinations of a Workcenter }\end{array}$ & Histogram of Process Time \\
\hline Throughput & $\begin{array}{l}\text { Show Throughput deviation per } \\
\text { Workcenter }\end{array}$ & $\begin{array}{l}\text { Throughput for all Equipment-EPA } \\
\text { combinations of a Workcenter }\end{array}$ & Histogram of Throughput \\
\hline Dedich Size & $\begin{array}{l}\text { Batch Size comparison (Max and } \\
\text { Average) per Workcenter }\end{array}$ & $\begin{array}{l}\text { Batch Size for Equipment belonged to } \\
\text { chosen Workcenter }\end{array}$ & $\begin{array}{l}\text { Histogram of Batch Size (Batch Size's } \\
\text { unit is number of Lot) }\end{array}$ \\
\hline Sampling Rate & $\begin{array}{l}\text { Show average number of incorrectly } \\
\text { assigned EPA per Workcenter }\end{array}$ & $\begin{array}{l}\text { List Additional and Missing EPAs for } \\
\text { every Equipment from picked } \\
\text { Workcenter }\end{array}$ & $\begin{array}{l}\text { Chart shows number of Moves for } \\
\text { each EPA of selected Equipment }\end{array}$ \\
\hline Stream Size & $\begin{array}{l}\text { Compare Sampling Rate for each } \\
\text { Workcenter }\end{array}$ & $\begin{array}{l}\text { Compare Sampling Rate for } \\
\text { combinations of Route and Operation in } \\
\text { appointed Workcenter }\end{array}$ & $\begin{array}{l}\text { Compare Sampling Rate for } \\
\text { combinations of Product and } \\
\text { Operation }\end{array}$ \\
\hline & $\begin{array}{l}\text { Display Stream Size at Workcenter } \\
\text { level }\end{array}$ & Display Stream Size per Equipment & $\begin{array}{l}\text { Histogram of Stream Size (Stream } \\
\text { Size's unit is number of Lot) }\end{array}$ \\
\hline
\end{tabular}

\section{AMV WORKFLOW}

Figure 5 illustrates the workflow in using the AMV module to detect and resolve data issues. The user verifies a past forecast period scenario and detects an issue with a modeling feature of a work center at Level 1 of AMV, for example a process time discrepancy for a work center that contributed to the WIP level discrepancy as shown in the gap between blue and green line of Figure 6 . The user then navigates to Level 2 to inspect the process time of equipment and process combinations for simulation and reality (Figure 7). At this level, the user is able to identify the specific equipment and processes that contribute towards the process time deviation. By navigating to Level 3, the user is able to look at the process time histogram for the specific equipment-process combination of simulation and reality. A corrected value can be derived from the histogram and be applied to the simulation model. The simulation model is executed with the corrected value, and an improvement is observed as illustrated in the red line of Figure 6. Following the same process, the AMV module can be used to quickly identify any data issues associated with modeling features and ensure that high forecast accuracy is maintained.

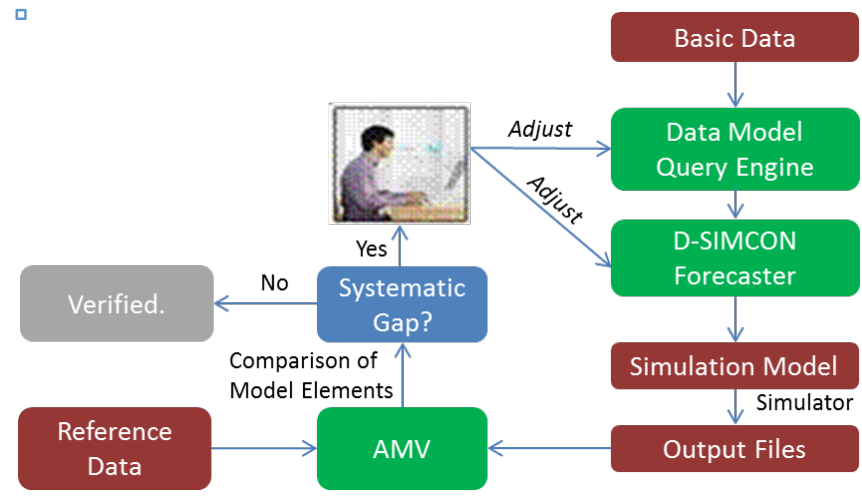

Figure 5: Using AMV to Identify Modeling Issues. 
Gan, Lendermann, Preuss, Scholl, and Mosinski

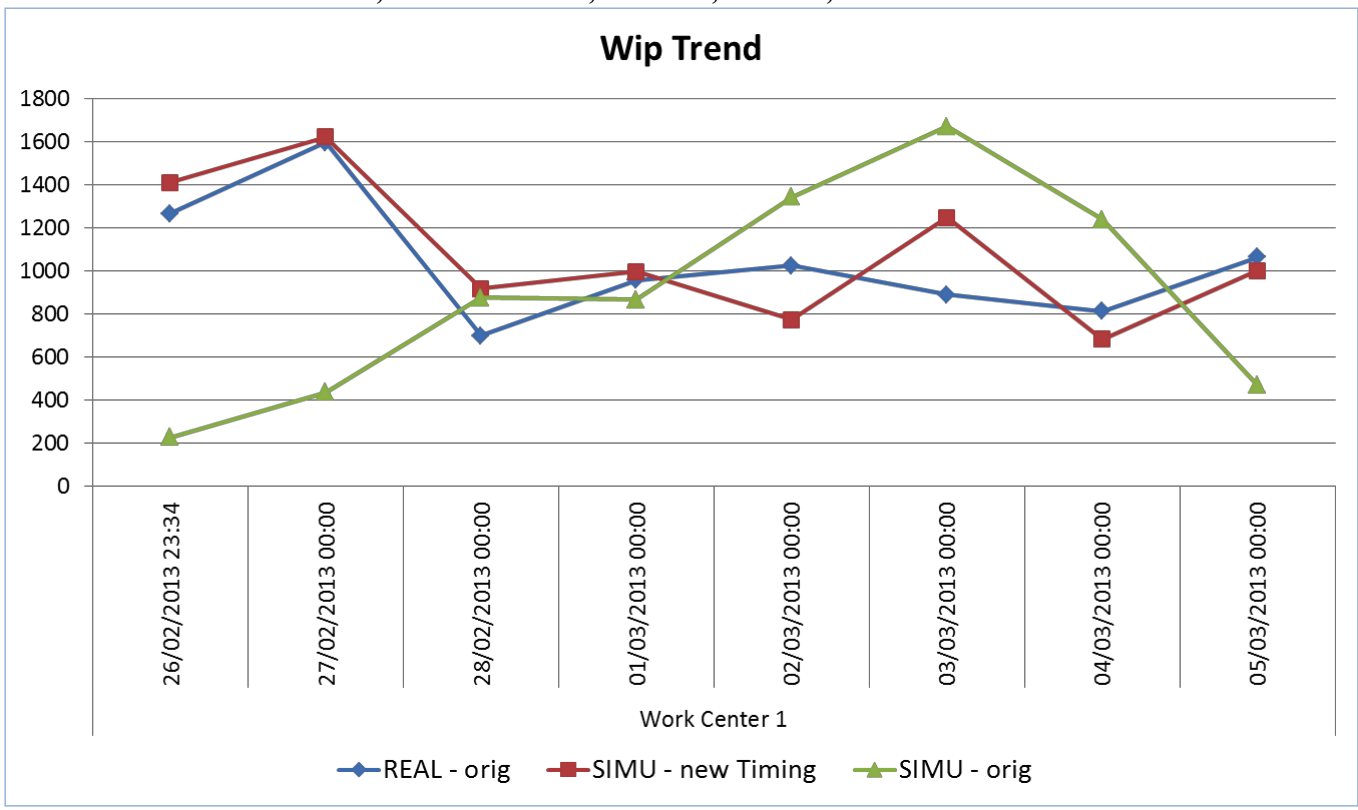

Figure 6: Improved WIP Trend through AMV-guided Model Enhancement.

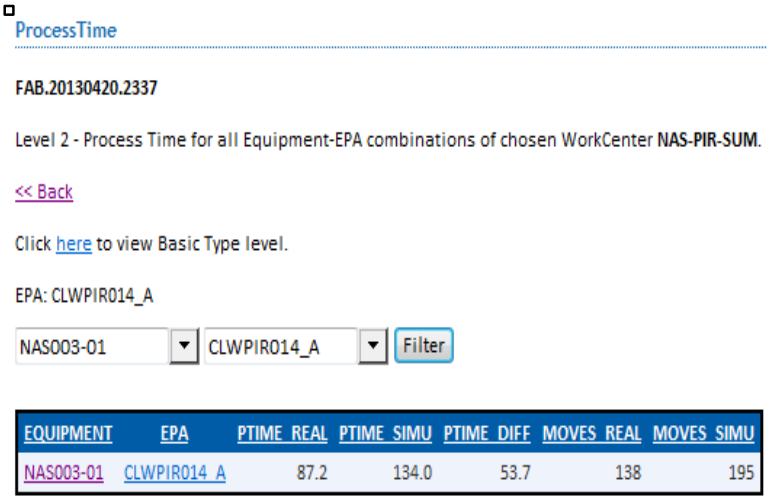

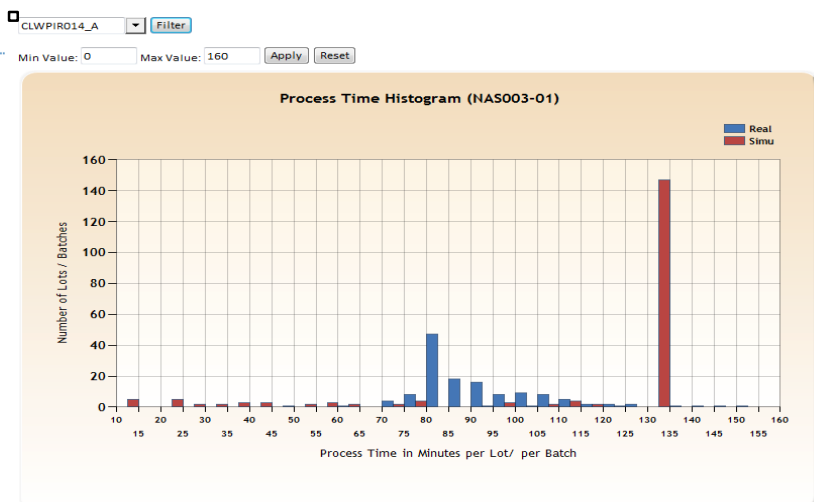

Figure 7: AMV Level 2 \& 3 - Visualization of Process Time Discrepancy.

\section{SUMMARY AND OUTLOOK}

In this paper we have shown how the approach of automated model verification is an important element to continuously monitor and verify simulation model to ensure the correctness of the forecast results. It enables the user to find basic data problems in a systematic manner through comparison of simulation data with historical real data. By visualizing the data discrepancies observed in three levels of granularity the method provides an easy and efficient way of resolving critical issues so as to maintain the forecast accuracy. The detection and correction of data issues is also communicated to the relevant department to enhance the quality of basic data.

Another application of AMV is to use it as an operational target evaluation tool. With a stable forecast accuracy, the expected key performance indicators (KPIs) for work center can be used as the operational target for the work center's operators. Any significant deviation between the expected and achieved KPIs value could mean that the operators did not adhere to the operational policies, such as dispatching decisions derive by a dispatching system. Using this approach, the operator can be educated on the importance of adherence to operational policies that gives global instead of local optimality operational results. 


\section{REFERENCES}

Gan, Lendermann, Preuss, Scholl, and Mosinski

D-SIMLAB, 2013. "D-SIMCON - An Overview". http://www.d-simlab.com/dsimcon.htm. Accessed April. 10th, 2013

Scholl, W., B.P. Gan, D. Noack, P. Preuss, M.L. Peh, P. Lendermann, and O. Rose. 2010. "Towards Realization of a High-Fidelity Simulation Model for Short-Term Horizon Forecasting in Wafer Fabrication Facilities." In Proceedings of the 2010 Winter Simulation Conference, edited by B. Johansson, S. Jain, J. Montoya-Torres, J. Hugan, and E. Yücesan, 2563-2574. Piscataway, New Jersey: Institute of Electrical and Electronics Engineers, Inc.

\section{AUTHOR BIOGRAPHIES}

BOON PING GAN is the CTO of D-SIMLAB Technologies (Singapore). He has been involved in simulation technology application and development since 1995, with primary focus on developing parallel and distributed simulation technology for complex systems such as semiconductor manufacturing and aviation spare inventory management. He was also responsible for several operations improvement projects with wafer fabrication clients which concluded with multi-million dollar savings. He is now responsible for managing the technology and product development at D-SIMLAB as well as execution of projects in the semiconductor and engineering domains. He holds a Master of Applied Science degree, specializing in Computer Engineering. His email address is boonping@d-simlab.com.

PETER LENDERMANN is the Co-Founder and CEO of D-SIMLAB Technologies, a Singapore-based company providing simulation-based decision support solutions and services to Aerospace, Semiconductor Manufacturing and other asset-intensive industries. Prior to this he worked at the Singapore Institute of Manufacturing Technology where he led the simulation-related research activities until spinning them off into D-SIMLAB Technologies. He has been engaged in the simulation community since the early 1990's. Peter holds a PhD in Applied High-Energy Physics from Humboldt University in Berlin (Germany) and an MBA in International Economics and Management from SDA Bocconi in Milan (Italy). His email address is peter@d-simlab.com.

PATRICK PREUSS is a Project Manager and the Deputy Manager Germany Operations of D-SIMLAB Technologies (Germany). He has been working in the development of simulation-based applications for Airbus, German Aerospace Centre and Infineon with focus on data analysis and heuristic optimization methods since 2005. Patrick holds a M.S. degree in computer science from Dresden University of Technology. His email address is patrick@d-simlab.com.

WOLFGANG SCHOLL works as a Senior Staff Expert for modeling and simulation for Infineon Technologies in Dresden (Germany). He has studied physics at the Technical University of Chemnitz (Germany) and graduated in solid-state physics in 1984. From 1984 to 1995 he worked as a process engineer for ZMD in Dresden. In 1996 he joined Infineon Technologies (former SIMEC) and worked on the field of capacity planning. Since 2003 he is responsible for fab simulation. His email address is wolfgang.scholl@infineon.com.

MARCIN MOSINSKI is a PhD student at Munich University of the Federal Armed Forces. He is a member of the scientific staff of Professor Oliver Rose at the Chair of Modeling and Simulation. He works as a Simulation Expert for a Short Term Simulation for Infineon Technologies in Dresden. He received his M.S. degree in computer science from Dresden University of Technology. His research interests include simulative and analytical forecasting of complex problems in manufacturing facilities and the statistical data analysis. His email address is marcin.mosinski@infineon.com. 